\title{
Optical Coherence Tomography Comparison of Percutaneous Coronary Intervention Among Plaque Rupture, Erosion, and Calcified Nodule in Acute Myocardial Infarction
}

Amir Kh. M. Khalifa, MD; Takashi Kubo, MD, PhD; Yasushi Ino, MD, PhD; Kosei Terada, MD; Hiroki Emori, MD; Daisuke Higashioka, MD; Yosuke Katayama, MD; Masahiro Takahata, MD; Kunihiro Shimamura, MD, PhD; Yasutsugu Shiono, MD, PhD; Yoshiki Matsuo, MD, PhD; Atsushi Tanaka, MD, PhD; Takeshi Hozumi, MD, PhD; Takashi Akasaka, MD, PhD

Background: Acute myocardial infarction (AMI) is caused by coronary plaque rupture (PR), plaque erosion (PE), or calcified nodule (CN). We used optical coherence tomography (OCT) to compare stent expansion immediately after primary percutaneous coronary intervention $(\mathrm{PCl})$ in patients with $\mathrm{AMl}$ caused by $\mathrm{PR}, \mathrm{PE}$, or $\mathrm{CN}$.

Methods and Results: In all, 288 AMI patients were assessed by OCT before and immediately after PCl, performed with OCT guidance according to OPINION criteria for stent sizing and optimization. The frequency of OCT-identified PR (OCT-PR), OCT-PE, and OCT-CN was $172(60 \%), 82(28 \%)$, and $34(12 \%)$, respectively. Minimum stent area was smallest in the OCT-CN group, followed by the OCT-PE and OCT-PR groups (mean $[ \pm S D] 5.20 \pm 1.77,5.44 \pm 1.78$, and $6.44 \pm 2.2 \mathrm{~mm}^{2}$, respectively; $P<0.001$ ), as was the stent expansion index $(76 \pm 13 \%, 86 \pm 14 \%$, and $87 \pm 16 \%$, respectively; $P=0.001)$. The frequency of stent malapposition was highest in the OCT-CN group, followed by the OCT-PR and OCT-PE groups $(71 \%, 38 \%$, and $27 \%$, respectively; $\mathrm{P}<0.001)$, as was the frequency of stent edge dissection in the proximal reference $(44 \%, 23 \%$, and $10 \%$, respectively; $\mathrm{P}<0.001)$. The frequency of tissue protrusion was highest in the OCT-PR group, followed by the OCT-PE and OCT-CN groups (95\%, $88 \%$, and $85 \%$, respectively; $\mathrm{P}=0.036$ ).

Conclusions: Stent expansion was smallest in the OCT-CN group, followed by the OCT-PR and OCT-PE groups. Plaque morphology in AMI culprit lesions may affect stent expansion immediately after primary PCl.

Key Words: Calcified nodule; Optical coherence tomography; Plaque erosion; Plaque rupture; Stent

A cute myocardial infarction (AMI) is caused by plaque rupture (PR), plaque erosion (PE), or calcified nodules $(\mathrm{CN})$ in coronary atherosclerotic lesions. Based on autopsy studies, PR is the most frequent etiology (55-60\%), followed by PE (30-35\%), with $\mathrm{CN}$ the least frequent etiology $(2-7 \%){ }^{1}$ PR is characterized by discontinuity in the fibrous cap and cavity formation within the lipid-rich plaque. ${ }^{1} \mathrm{PE}$ is characterized by an area lacking surface endothelium and occurs over a plaque with a thick intima. ${ }^{1} \mathrm{CN}$ is a plaque with luminal thrombi showing calcific nodules protruding into the lumen through a disrupted fibrous cap. ${ }^{1}$

Percutaneous coronary intervention (PCI) using stents is established therapy for coronary artery disease. Stent expansion is closely associated with PCI outcomes. Previous intravascular imaging studies have demonstrated that stent underexpansion is a major predictor of subsequent stent failure, such as restenosis and thrombosis. ${ }^{2,3}$ Stent expansion is potentially affected by underlying lesion mor- phologies and plaque tissue components.

Intracoronary optical coherence tomography (OCT) allows PR, PE, and CN to be differentiated in vivo, as well as stent expansion to be assessed during PCI. ${ }^{4}$ The present study used OCT to compare stent expansion immediately after primary $\mathrm{PCI}$ in AMI caused by PR, $\mathrm{PE}$, and $\mathrm{CN}$.

\section{Study Population}

\section{Methods}

This study was a retrospective in AMI patients who underwent PCI with OCT guidance between January 2016 and December 2018 at Wakayama Medical University, Wakayama, Japan. Based on OCT findings in the AMI culprit lesions before $\mathrm{PCI}$, patients were categorized into 3 groups (i.e., OCT-PR, OCT-PE, or OCT-CN), with stent expansion immediately after PCI compared among the 3 groups. AMI was defined according to the universal definition. ${ }^{5}$ Patients with AMI due to vasospasm, coronary embo-

Received January 26, 2020; revised manuscript received February 28, 2020; accepted March 11, 2020; J-STAGE Advance Publication released online April 18, 2020 Time for primary review: 23 days

Department of Cardiovascular Medicine, Wakayama Medical University, Wakayama, Japan

Mailing address: Takashi Kubo, MD, PhD, FJCS, Department of Cardiovascular Medicine, Wakayama Medical University, 811-1 Kimiidera, Wakayama 641-8509, Japan. E-mail: takakubo@wakayama-med.ac.jp

ISSN-1346-9843 All rights are reserved to the Japanese Circulation Society. For permissions, please e-mail: cj@j-circ.or.jp 

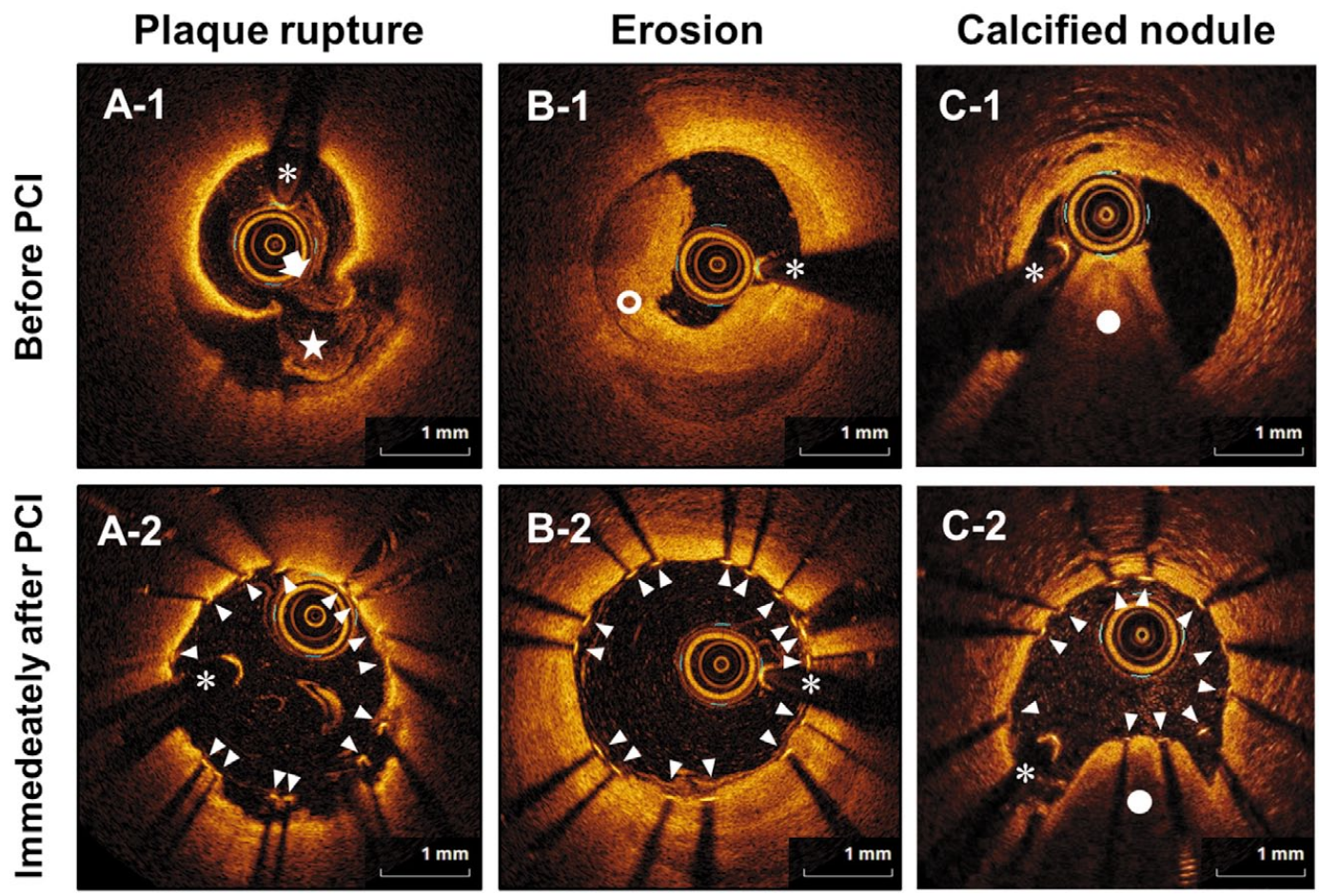

Figure 1. Representative optical coherence tomography (OCT) images. OCT shows $(\mathbf{A - 1}, \mathbf{2})$ plaque rupture, $(\mathbf{B}-\mathbf{1}, \mathbf{2})$ erosion, and $(\mathbf{C}-\mathbf{1}, \mathbf{2})$ calcified nodule before and immediately after percutaneous coronary intervention $(\mathrm{PCI})$. The arrow indicates a disrupted fibrous cap, arrowheads indicate stent struts, asterisks indicate the guidewire, closed circles indicate calcium, the open circle indicates a thrombus, and the star indicates the plaque cavity.

lization, spontaneous coronary artery dissection, stent thrombosis, or coronary artery bypass graft failure were excluded from the study. Patients who had no stent implantation or inadequate OCT before and/or immediately after PCI were also excluded from the analysis.

This study was approved by the institutional review board of Wakayama Medical University Ethics Committee (Reference no. 1920). The requirement for written informed consent was waived because of the retrospective design of the study.

\section{Patient Clinical Characteristics}

Patient clinical data were collected by reviewing all available medical records. Patient clinical data included age, sex, hypertension (defined as systolic blood pressure $\geq 140 \mathrm{mmHg}$, diastolic blood pressure $\geq 90 \mathrm{mmHg}$, or the use of antihypertensive medication), diabetes (defined as $\mathrm{HbA} 1 \mathrm{c} \geq 6.5 \%$ or the use of antidiabetic medication), dyslipidemia (defined as low-density lipoprotein cholesterol $\geq 140 \mathrm{mg} / \mathrm{dL}$ or the use of antidyslipidemic medication), current smoking status (defined as having smoked at least 100 cigarettes over the lifetime and smoking currently), a family history of coronary artery disease (defined as the presence of angina, myocardial infarction [MI], or coronary revascularization in a first-degree relative), obesity (defined as a body mass index $\geq 25 \mathrm{~kg} / \mathrm{m}^{2}$ ), hemodialysis, chronic kidney disease with an estimated glomerular filtration rate (eGFR) $<30 \mathrm{~mL} / \mathrm{min} / 1.73 \mathrm{~m}^{2}$, and prior $\mathrm{MI}$.

\section{Angiographic and Procedural Characteristics}

Angiograms were reviewed by an investigator (Y.I.). The culprit lesion of AMI was identified by angiographic lesion morphology such as total or subtotal occlusion as well as electrocardiographic findings and echocardiographic results. Angiographic data included the culprit vessels, the location of the culprit lesion, and TIMI flow grade (i.e., 0, $1,2$ or 3$).^{6}$

PCI was performed with OCT guidance according to the OPtical frequency domain imaging vs. INtravascular ultrasound in percutaneous coronary InterventiON (OPINION) criteria for stent sizing and optimization..$^{7-9}$ All PCI operators were educated about the OPINION criteria, and during the procedure at least one experienced OCT specialist provided guidance for the PCI based on the OPINION criteria. PCI procedural data were collected by reviewing medical records. PCI procedural data included thrombus aspiration, stent type, stent size, stent length, post-dilatation, maximum balloon diameter (including stent balloon), balloon-to-artery ratio (defined as maximum balloon diameter divided by OCT-measured mean lumen diameter at the proximal reference), and maximum inflation pressure.

\section{OCT}

OCT was performed by using either ILUMIEN OPTIS (Abbott Vascular, Santa Clara, CA, USA) or LUNAWAVE (Terumo, Tokyo, Japan). In cases with TIMI flow Grade 0 or 1 , aspiration thrombectomy was performed prior to OCT imaging. The OCT catheter was advanced distally to the culprit lesion over a 0.014 -inch conventional angioplasty 


\begin{tabular}{|c|c|c|c|c|}
\hline & $\begin{array}{l}\text { OCT-PR } \\
(n=172)\end{array}$ & $\begin{array}{c}\text { OCT-PE } \\
(n=82)\end{array}$ & $\begin{array}{c}\text { OCT-CN } \\
(n=34)\end{array}$ & P-value \\
\hline Age (years) & $70.0 \pm 12.3$ & $67.9 \pm 13.7$ & $77.4 \pm 10.7^{\star, \dagger}$ & 0.001 \\
\hline Male sex & $131(76)$ & $54(66)$ & $15(44)^{\dagger}$ & 0.001 \\
\hline \multicolumn{5}{|l|}{ Risk factors } \\
\hline Hypertension & $145(84)$ & $64(78)$ & $33(97)^{*}$ & 0.039 \\
\hline Diabetes & $69(40)$ & $27(33)$ & $17(50)$ & 0.215 \\
\hline Dyslipidemia & $135(78)$ & $59(72)$ & $25(74)$ & 0.488 \\
\hline Current smoking & $50(29)$ & $35(43)$ & $5(15)^{*}$ & 0.008 \\
\hline Family history of CAD & $21(12)$ & $18(22)$ & $5(15)$ & 0.130 \\
\hline Obesity & $40(23)$ & $21(26)$ & $10(29)$ & 0.798 \\
\hline Hemodialysis & $5(3)$ & $2(2)$ & $4(12)$ & 0.036 \\
\hline CKD $\left(\right.$ eGFR $\left.<30 \mathrm{~mL} / \mathrm{min} / 1.73 \mathrm{~m}^{2}\right)$ & $11(6)$ & $4(6)$ & $5(15)$ & 0.150 \\
\hline Prior Ml & $10(6)$ & $2(2)$ & $4(12)$ & 0.133 \\
\hline \multicolumn{5}{|l|}{ Clinical presentation } \\
\hline STEMI & $138(80)$ & $62(76)$ & $22(65)$ & 0.134 \\
\hline NSTEMI & $33(19)$ & $20(24)$ & $12(35)$ & 0.109 \\
\hline
\end{tabular}

Values are presented as $\mathrm{n}(\%)$ or as the mean $\pm \mathrm{SD}$ for patients with optical coherence tomography (OCT)-identified, plaque rupture, plaque erosion, and calcified nodules (OCT-PR, OCT-PE, and OCT-CN, respectively). ${ }^{*}<0.0167$ for OCT-PE vs. OCT-CN; ${ }^{P}<0.0167$ for OCT-CN vs. OCT-PR. CAD, coronary artery disease; CKD, chronic kidney disease; eGFR, estimated glomerular filtration rate; MI, myocardial infarction; NSTEMI, non-ST elevation myocardial infarction; STEMI, ST elevation myocardial infarction.

\begin{tabular}{|c|c|c|c|c|}
\hline & $\begin{array}{l}\text { OCT-PR } \\
(n=172)\end{array}$ & $\begin{array}{c}\text { OCT-PE } \\
(n=82)\end{array}$ & $\begin{array}{c}\text { OCT-CN } \\
(n=34)\end{array}$ & P-value \\
\hline \multicolumn{5}{|l|}{ Culprit artery } \\
\hline LAD/LCX/RCA & $84 / 18 / 70$ & $43 / 12 / 27$ & $21 / 4 / 9$ & 0.434 \\
\hline \multicolumn{5}{|l|}{ Lesion location } \\
\hline Proximal/mid/distal & $83 / 63 / 26$ & $38 / 31 / 13$ & $18 / 14 / 2$ & 0.683 \\
\hline \multicolumn{5}{|l|}{ TIMI flow grade } \\
\hline 0 or 1 & $97(56)$ & $46(56)$ & $10(29)^{t, \ddagger}$ & 0.013 \\
\hline \multicolumn{5}{|l|}{$\mathrm{PCl}$ procedure } \\
\hline Thrombus aspiration & $66(38)$ & $31(43)$ & $5(15)^{\ddagger}$ & 0.027 \\
\hline Stent diameter $(\mathrm{mm})$ & $3.23 \pm 0.46^{*}$ & $3.06 \pm 0.46$ & $3.02 \pm 0.47^{\ddagger}$ & 0.005 \\
\hline Stent length $(\mathrm{mm})$ & $23 \pm 7$ & $22 \pm 7$ & $23 \pm 8$ & 0.442 \\
\hline Stent type & & & & 0.779 \\
\hline XIENCE stent & $84(49)$ & $46(56)$ & $15(44)$ & \\
\hline Synergy stent & $42(24)$ & $16(20)$ & $11(32)$ & \\
\hline Ultimaster stent & $37(22)$ & $18(22)$ & $6(18)$ & \\
\hline Orsiro stent & $4(2)$ & $1(1)$ & $0(0)$ & \\
\hline Resolute stent & $2(1)$ & $1(1)$ & $1(3)$ & \\
\hline Bare-metal stent & $3(2)$ & $0(0)$ & $1(3)$ & \\
\hline Post-dilatation & $110(64)$ & $48(56)$ & $29(85)$ & 0.053 \\
\hline Maximum balloon diameter $(\mathrm{mm})$ & $3.43 \pm 0.54^{*}$ & $3.16 \pm 0.51$ & $3.20 \pm 0.48$ & $<0.001$ \\
\hline Balloon-to-artery ratio & $1.07 \pm 0.17$ & $1.08 \pm 0.14$ & $1.06 \pm 0.13$ & 0.801 \\
\hline Maximum inflation pressure (atm) & $16 \pm 4$ & $16 \pm 4$ & $18 \pm 4$ & 0.099 \\
\hline
\end{tabular}

Values are presented as $\mathrm{n}(\%)$ or as the mean \pm SD for patients with optical coherence tomography (OCT)-identified, plaque rupture, plaque erosion, and calcified nodules (OCT-PR, OCT-PE, and OCT-CN, respectively). ${ }^{*}<<0.0167$ for OCT-PR vs. OCT-PE; ${ }^{\mathrm{P}} \mathrm{P}<0.0167$ for OCT-PE vs. OCT-CN; ${ }^{\mathrm{P}}<0.0167$ for OCT-CN vs. OCT-PR. LAD, left anterior descending artery; LCX, left circumflex artery; $\mathrm{PCl}$, percutaneous coronary intervention; RCA, right coronary artery; TIMI, Thrombolysis in Myocardial Infarction.

guidewire. The OCT images were acquired during intracoronary contrast injection and automatic catheter pullback.

The OCT analysis was performed using dedicated offline review systems (Abbott Vascular or Terumo). The OCT images obtained before PCI were analyzed by an experi- enced investigator (T.K.). Underling plaque morphology was evaluated in every frame throughout the culprit lesion. OCT-PR was defined as fibrous cap disruption with a clear cavity formed inside the plaque; OCT-PE was defined as the presence of an attached thrombus overlying an intact 


\begin{tabular}{|c|c|c|c|c|}
\hline & $\begin{array}{l}\text { OCT-PR } \\
(n=172)\end{array}$ & $\begin{array}{c}\text { OCT-PE } \\
(n=82)\end{array}$ & $\begin{array}{c}\text { OCT-CN } \\
(n=34)\end{array}$ & P-value \\
\hline \multicolumn{5}{|l|}{ Proximal reference } \\
\hline Lumen area $\left(\mathrm{mm}^{2}\right)$ & $8.68 \pm 3.29^{*}$ & $7.23 \pm 2.83$ & $7.67 \pm 3.19$ & 0.002 \\
\hline Dissection & $39(23)^{*}$ & $8(10)$ & $15(44)^{\dagger, \ddagger}$ & $<0.001$ \\
\hline \multicolumn{5}{|l|}{ Distal reference } \\
\hline Lumen area $\left(\mathrm{mm}^{2}\right)$ & $6.48 \pm 2.49^{*}$ & $5.64 \pm 2.28$ & $6.12 \pm 2.58$ & 0.040 \\
\hline Dissection & $46(27)$ & $14(17)$ & $10(29)$ & 0.190 \\
\hline \multicolumn{5}{|l|}{ Stent segment } \\
\hline Minimum stent area $\left(\mathrm{mm}^{2}\right)$ & $6.44 \pm 2.20^{*}$ & $5.44 \pm 1.78$ & $5.20 \pm 1.77^{\ddagger}$ & $<0.001$ \\
\hline Stent expansion index (\%) & $86 \pm 14$ & $87 \pm 16$ & $76 \pm 13^{\dagger, \ddagger}$ & $<0.001$ \\
\hline Minimum lumen area $\left(\mathrm{mm}^{2}\right)$ & $5.71 \pm 1.91^{*}$ & $5.03 \pm 1.72$ & $5.01 \pm 1.93$ & 0.013 \\
\hline Stent malapposition & $66(38)$ & $22(27)$ & $24(71)^{\dagger, \neq}$ & $<0.001$ \\
\hline Tissue protrusion & $164(95)$ & $72(88)$ & $29(85)$ & 0.036 \\
\hline Irregular tissue protrusion & $158(92)$ & $68(77)$ & $20(59)^{\dagger, \neq}$ & $<0.001$ \\
\hline
\end{tabular}

Values are presented as number (\%) or mean \pm SD for patients with optical coherence tomography (OCT)-identified, plaque rupture, plaque erosion, and calcified nodules (OCT-PR, OCT-PE, and OCT-CN, respectively). ${ }^{*} \mathrm{P}<0.0167$ for OCT-PR vs. OCT-PE; †P<0.0167 for OCT-PE vs. OCT-CN; $¥$ P $<0.0167$ for OCT-CN vs. OCT-PR.

and visualized plaque, luminal surface irregularity at the culprit lesion in the absence of thrombus, or attenuation of the underlying plaque by a thrombus without superficial lipid or calcification immediately proximal or distal to the site of the thrombus; and OCT-CN was defined as fibrous cap disruption detected over a calcified plaque characterized by protruding calcification, superficial calcium, and/ or the presence of substantive calcium proximal and/or distal to the lesion. ${ }^{4}$ Inter- and intra-observer variability were assessed by the evaluation of 30 randomly selected images by 2 independent observers and by the same observer at 2 separate time points, respectively. The interobserver $\kappa$ coefficients for OCT-PR, OCT-PE, and OCT-CN were $0.93,0.92$, and 1.00 , respectively. The intra-observer $\kappa$ coefficients for OCT-PR, OCT-PE, and OCT-CN were 0.92 , 0.93 , and 1.00 , respectively.

The OCT images immediately after PCI were analyzed by an investigator (A.K.) who was blinded to the OCT findings before PCI. Lumen and stent areas were measured in every frame. The OCT analysis immediately after PCI included lumen areas at the proximal and distal references (defined as the frames just proximal or distal to stent edges), minimum lumen areas in the stent segment, minimum stent area, and stent expansion index (defined as minimum stent area divided by the average of the proximal and distal reference lumen areas multiplied 100). Dissection was defined as disruption of the luminal vessel surface at the stent edges, stent malapposition was defined as incomplete stent strut apposition $>0.35 \mathrm{~mm}$ with longitudinal extension $>1 \mathrm{~mm}$, and tissue protrusion was defined as tissue prolapse between adjacent stent struts towards the lumen.

\section{Statistical Analysis}

Statistical comparisons among the OCT-PR, OCT-PE, and OCT-CN groups were performed using JMP 13.0 (SAS Institute, Cary, NC, USA). Categorical variables are presented as numbers and percentages and were compared among the 3 groups by the Chi-squared or Fisher's exact test (for an expected cell value $<5$ ) with pairwise tests using the Bonferroni correction for multiple comparisons. Continuous variables are presented as the mean $\pm \mathrm{SD}$ and were compared among the 3 groups by factorial analysis of variance
(ANOVA) with post hoc analysis using the Bonferroni correction for multiple pairwise comparisons. A P-values two-sided $<0.05$ was considered statistically significant in comparisons among the 3 groups. In the post hoc analysis using the Bonferroni correction for multiple pairwise comparisons, significance was set at $\mathrm{P}<0.0167(=0.05 / 3)$.

\section{Results}

In all, 373 patients with AMI were identified during the study period. Of these patients, $325(87 \%)$ had undergone PCI with OCT guidance. Thirty-seven patients were subsequently excluded from the analysis because of no stent implantation $(n=5)$, AMI due to stent thrombosis $(n=7)$, or inadequate OCT before and/or immediately after PCI $(n=25)$. Thus, the final study population consisted of 288 patients. In this study population, the frequency of OCTPR, OCT-PE, and OCT-CN was $172(60 \%), 82(28 \%)$, and $34(12 \%)$, respectively. Representative images of OCT-PR, OCT-PE, and OCT-CN are shown in Figure 1.

Patient clinical characteristics are presented in Table 1. There was a significant difference in age $(\mathrm{P}=0.001)$, sex $(\mathrm{P}=0.001)$, hypertension $(\mathrm{P}=0.039)$, current smoking $(\mathrm{P}=0.008)$, and hemodialysis $(\mathrm{P}=0.036)$, but not in diabetes, dyslipidemia, a family history of coronary artery disease, obesity, chronic kidney disease with eGFR $<30 \mathrm{~mL} /$ $\mathrm{min} / 1.73 \mathrm{~m}^{2}$, and prior MI, among the OCT-PR, OCT-PE, and OCT-CN groups. The frequency of ST-elevation myocardial infarction (STEMI) and non-STEMI (NSTEMI) was similar among the 3 groups.

Angiographic and procedural characteristics are summarized in Table 2. The culprit coronary artery and the location of the culprit lesion were similar among the OCTPR, OCT-PE, and OCT-CN groups. The frequency of TIMI flow Grade 0 or 1 differed significantly among the 3 groups $(\mathrm{P}=0.013)$. Although the frequency of thrombus aspiration, stent diameter, and maximum balloon diameter differed significantly among the 3 groups $(\mathrm{P}=0.027, \mathrm{P}=0.005$, and $\mathrm{P}<0.001$, respectively), there were no significant differences in stent type, stent length, post-dilatation, balloonto-artery ratio, and maximum inflation pressure among the 3 groups. 
The OCT findings are summarized in Table 3. Minimum stent area was smallest in the OCT-CN group $\left(5.20 \pm 1.77 \mathrm{~mm}^{2}\right)$, followed by the OCT-PE and OCT-PR groups $(5.44 \pm 1.78$ and $6.44 \pm 2.20 \mathrm{~mm}^{2}$ ), respectively; $\mathrm{P}<0.001$ ). Reference lumen areas were significantly different among the 3 groups: largest in the OCT-PR group, followed by the OCT-CN and OCT-PE groups (proximal reference: $8.68 \pm 3.29$ vs. $7.67 \pm 3.19$ and $7.23 \pm 2.83 \mathrm{~mm}^{2}$, respectively $[\mathrm{P}=0.002]$; distal reference: $6.48 \pm 2.49$ vs. $6.12 \pm 2.58$ and $5.64 \pm 2.28 \mathrm{~mm}^{2}$, respectively $\left.[\mathrm{P}=0.040]\right)$. The stent expansion index was smallest in the OCT-CN group $(76 \pm 13 \%)$, followed by the OCT-PR and OCT-PE groups $(86 \pm 14 \%$ and $87 \pm 16 \%$, respectively; $\mathrm{P}<0.001$; Figure 2). Minimum lumen area in the stent segment was smallest in the OCTCN group $\left(5.01 \pm 1.93 \mathrm{~mm}^{2}\right)$, followed by the OCT-PE and OCT-PR groups $\left(5.03 \pm 1.72\right.$ and $5.71 \pm 1.91 \mathrm{~mm}^{2}$, respectively; $\mathrm{P}=0.013$ ). The frequency of stent malapposition was highest in the OCT-CN group (71\%), followed by the OCT-PR and OCT-PE groups (38\% and 27\%, respectively; $\mathrm{P}<0.001)$. The frequency of tissue protrusion was highest in the OCT-PR group (95\%), followed by the OCT-PE and OCT-CN groups $(88 \%$ and $85 \%$, respectively; $\mathrm{P}=0.036)$. The frequency of irregular tissue protrusion was also highest in the OCT-PR group (92\%), followed by the OCT-PE and OCT-CN groups (77\% and 59\%, respectively; $\mathrm{P}<0.001$ ). The frequency of stent edge dissection in the proximal reference was highest in the OCT-CN group (44\%), followed by the OCT-PR and OCT-PE groups $(23 \%$ and $10 \%$, respectively; $\mathrm{P}<0.001$ ), but there was no difference in the frequency of stent edge dissection in the distal reference among the 3 groups.

\section{Discussion}

The main finding of the present study was that stent expansion was smallest in the OCT-CN group, followed by the OCT-PR and OCT-PE groups. The plaque morphology in AMI culprit lesions may affect stent expansion immediately after primary PCI.

\section{$\mathrm{PCI}$ in PR, PE, and CN}

To date, no studies have directly compared the long-term clinical prognosis of patients with primary PCI in AMI according to the presence of PR, PE, or CN. ${ }^{10}$ Only one study, by Higuma et al, compared acute results of primary PCI among PR, PE, and CN groups. ${ }^{11}$ That study analyzed coronary angiography immediately after primary PCI and showed that the minimum lumen diameter was numerically smallest in the $\mathrm{CN}$ group and that the percentage diameter stenosis was numerically greatest in this group. ${ }^{11}$ However, that study had no OCT data immediately after primary PCI. Therefore, in the present study we used OCT to investigate the acute results of primary PCI among the PR, PE, and $\mathrm{CN}$ groups. To the best of our knowledge, the present study is the first to demonstrate, using OCT, smallest stent expansion immediately after primary PCI in the presence of $\mathrm{CN}$.

\section{Stent Underexpansion}

Stent underexpansion is the most powerful predictor of long-term stent patency and clinical outcome. The Centro per la Lotta contro l'Infarto-Optimisation of Percutaneous Coronary Intervention (CLI-OPCI) study of OCT during PCI showed that stent underexpansion, defined as a minimum stent area $<4.5 \mathrm{~mm}^{2}$, was responsible for the majority

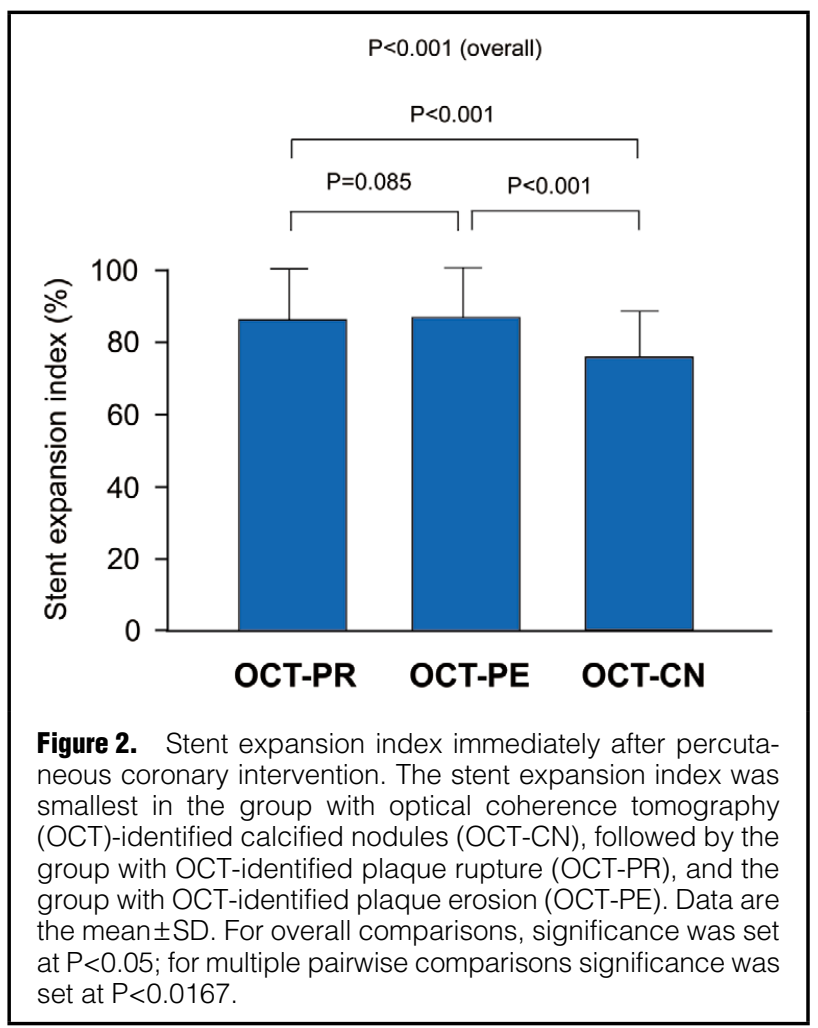

of stent restenoses. ${ }^{12}$ The PREvention of Stent Thrombosis by an Interdisciplinary Global European Effort (PRESTIGE) study of OCT in stent thrombosis demonstrated that stent underexpansion, defined as a stent expansion index $<0.8$, was the most dominant finding in acute and subacute stent thrombosis. ${ }^{13}$ Based on this evidence, the present study suggests that $\mathrm{CN}$, with the smallest stent expansion, may be associated with the highest risk of stent restenosis and thrombosis compared with PR and PE.

\section{Aggressive Lesion Preparation}

Achieving sufficient stent expansion in $\mathrm{CN}$ is a considerable challenge. In $\mathrm{CN}$, similar to stable severe calcified lesions, predilatation with a high-pressure balloon, a large-sized balloon, or a cutting balloon may help break superficial calcifications and achieve sufficient stent expansion. ${ }^{14}$ Excimer laser coronary angioplasty, which can be used for thrombotic lesions, may be an alternative option for lesion preparation..$^{15}$ When lesion preparation is inadequate, avoiding stent implantation should be considered. Following a certain period of antiplatelet therapy, rotational atherectomy may be effective. ${ }^{16}$ OCT is useful for identifying lesions that require aggressive lesion preparation before stent implantation, and the OCT guidance effects a significant change in PCI strategies in AMI.

\section{Study Limitations}

The present study has several limitations. First, the present study was a single-center retrospective non-randomized study, which may carry selection bias. However, OCT was performed in $87 \%$ of all-comers with AMI during the study period who were enrolled in the study. Second, the ability of OCT to diagnose PR, PE, or CN decreases in the presence of a large thrombus burden, especially for patients 
with AMI. To overcome this issue, we performed aspiration thrombectomy prior to OCT imaging when the thrombi completely or almost completely occluded the lumen. Third, the number of PE and CN was small. However, the results of this study are in agreement with those of previous autopsy studies ${ }^{1}$ showing a low incidence of PE and CN. Fourth, in contrast with previous results reported by Jia et $\mathrm{al},{ }^{4}$ there was no significant difference in the proportion of STEMI and NSTEMI among the OCT-PR, OCT-PE, and OCT-CN groups in the present study and the distribution of culprit coronary arteries and the location of culprit lesions were similar among the 3 groups. Unlike in the study of Jia et al, ${ }^{4}$ in the present study we excluded patients with unstable angina from analysis. This may have led to differences in patient clinical and angiographic characteristics between the previous and present studies. Fifth, in the $\mathrm{CN}$ group, the size and hardness of the protruding $\mathrm{CN}$ and the extent of the underlying calcification may affect stent expansion. Individual differences in the degree of stent expansion need to be investigated in a further study with a larger number of patients with $\mathrm{CN}$. Finally, this study has no data on long-term clinical outcomes. A further study is needed in a larger study population with long-term follow-up.

\section{Conclusions}

Stent expansion immediately after primary PCI was different among the OCT-PR, OCT-PE, and OCT-CN groups. Stent expansion was smallest in the OCT-CN group. Plaque morphology in AMI culprit lesions may affect stent expansion immediately after primary PCI.

\section{Sources of Funding}

None.

\section{Disclosures}

T.A. is a member of Circulation Journal' Editorial Team.

\section{Conflict of Interest}

T.A. has received lecture fees and research grants from Abbott and Terumo. T.K. has received lecture fees from Abbott. All other authors have no relationships relevant to the contents of this paper to disclose.

\section{References}

1. Virmani R, Kolodgie FD, Burke AP, Farb A, Schwartz SM. Lessons from sudden coronary death: A comprehensive morphological classification scheme for atherosclerotic lesions. Arterioscler Thromb Vasc Biol 2000; 20: 1262-1275.

2. Sonoda S, Morino Y, Ako J, Terashima M, Hassan AH, Bonneau $\mathrm{HN}$, et al. Impact of final stent dimensions on long-term results following sirolimus-eluting stent implantation: Serial intravascular ultrasound analysis from the sirius trial. $J$ Am Coll Cardiol 2004; 43: 1959-1963.

3. Morino Y, Honda Y, Okura H, Oshima A, Hayase M, Bonneau $\mathrm{HN}$, et al. An optimal diagnostic threshold for minimal stent area to predict target lesion revascularization following stent implantation in native coronary lesions. Am J Cardiol 2001; 88: 301-303.

4. Jia H, Abtahian F, Aguirre AD, Lee S, Chia S, Lowe H, et al. In vivo diagnosis of plaque erosion and calcified nodule in patients with acute coronary syndrome by intravascular optical coherence tomography. J Am Coll Cardiol 2013; 62: 1748-1758.

5. Thygesen K, Alpert JS, Jaffe AS, Chaitman BR, Bax JJ, Morrow DA, et al. Fourth universal definition of myocardial infarction (2018). J Am Coll Cardiol 2018; 72: 2231-2264.

6. TIMI Study Group. The Thrombolysis in Myocardial Infarction (TIMI) trial: Phase I findings. N Engl J Med 1985; 312: 932-936.

7. Kubo T, Shinke T, Okamura T, Hibi K, Nakazawa G, Morino $Y$, et al. Optical frequency domain imaging vs. intravascular ultrasound in percutaneous coronary intervention (OPINION trial): Study protocol for a randomized controlled trial. J Cardiol 2016; 68: $455-460$.

8. Kubo T, Shinke T, Okamura T, Hibi K, Nakazawa G, Morino $\mathrm{Y}$, et al. Optical frequency domain imaging versus intravascular ultrasound in percutaneous coronary intervention (OPINION trial): 1-year angiographic and clinical results. Eur Heart $J$ 2017; 38: $3139-3147$.

9. Otake H, Kubo T, Takahashi H, Shinke T, Okamura T, Hibi K, et al. Optical frequency domain imaging versus intravascular ultrasound in percutaneous coronary intervention (OPINION trial): Results from the OPINION imaging study. JACC Cardiovasc Imaging 2018; 11: 111-123.

10. Yonetsu T, Lee T, Murai T, Suzuki M, Matsumura A, Hashimoto Y, et al. Plaque morphologies and the clinical prognosis of acute coronary syndrome caused by lesions with intact fibrous cap diagnosed by optical coherence tomography. Int J Cardiol 2016; 203: $766-774$.

11. Higuma T, Soeda T, Abe N, Yamada M, Yokoyama H, Shibutani $\mathrm{S}$, et al. A combined optical coherence tomography and intravascular ultrasound study on plaque rupture, plaque erosion, and calcified nodule in patients with ST-segment elevation myocardial infarction: Incidence, morphologic characteristics, and outcomes after percutaneous coronary intervention. JACC Cardiovasc Interv 2015; 8: 1166-1176.

12. Prati F, Romagnoli E, Burzotta F, Limbruno U, Gatto L, La Manna A, et al. Clinical impact of OCT findings during PCI. The CLI-OPCI II Study. JACC Cardiovasc Imaging 2015; 8: 1297 1305 .

13. Adriaenssens T, Joner M, Godschalk TC, Malik N, Alfonso F, Xhepa E, et al. Optical coherence tomography findings in patients with coronary stent thrombosis: A report of the PRESTIGE Consortium (Prevention of Late Stent Thrombosis by an Interdisciplinary Global European Effort). Circulation 2017; 136: 1007-1021.

14. Kobayashi Y, Okura H, Kume T, Yamada R, Kobayashi Y, Fukuhara K, et al. Impact of target lesion coronary calcification on stent expansion. Circ J 2014; 78: 2209-2214.

15. Ashikaga T, Yoshikawa S, Isobe M. The efficacy of excimer laser pretreatment for calcified nodule in acute coronary syndrome. Cardiovasc Revasc Med 2015; 16: 197-200.

16. Kobayashi N, Ito Y, Yamawaki M, Araki M, Sakai T, Sakamoto $\mathrm{Y}$, et al. Distal embolization of coronary calcified nodule after rotational atherectomy. SAGE Open Med Case Rep 2018; 6: 1-5. 